\title{
Le jeu des contraintes dans la sélection du thème présuffixal
}

\author{
Roché, Michel, \& Plénat, Marc \\ ERSS (CH) / Université Toulouse-Le Mirail - mroche@univ-tlse2.fr \\ ERSS (CH) / CLVA - plenat@univ-tlse2.fr
}

\begin{abstract}
Dans le cadre d'une morphologie lexématique, les notions de thème (Aronoff, 1994, 2012) et d'« espace thématique » (Bonami et Boyé, 2003, 2005, entre autres) ont fait leurs preuves pour rendre compte de l'allomorphie radicale des lexèmes en ce qui concerne la flexion. Leurs premières applications à la dérivation (Plénat, 2008, 2009 ; Bonami, Boyé et Kerleroux, 2009; Villoing, 2009 ; Tribout, 2012 ; Boyé et Plénat, à paraître) ont également été prometteuses : chaque type de dérivation, comme chaque tiroir de la flexion, semblait sélectionner toujours le même thème. Des difficultés, cependant, n'ont pas tardé à apparaître. Roché (2010) a proposé de distinguer les notions de thème et de radical, plus ou moins confondues dans les travaux concernant la flexion, où cette distinction n'est pas nécessaire. Plénat et Roché (sous presse), après Plénat (2008) ont montré que le thème « savant » d'un adjectif ou d'un nom pouvait prendre plusieurs formes pour un même lexème, et que le choix de l'une ou l'autre de ces formes obéissait à un certain nombre de contraintes, d'ordre lexical ou phonologique. Roché (2013) aboutit à la même conclusion pour le thème présuffixal "populaire ». Cette « overabundance» (Thornton, 2012) n'est pas sans poser problème, puisque l'un des deux principes de base, dans cette approche de l'allomorphie radicale, est que chaque case de l'espace thématique d'un lexème contient une seule forme. Ces travaux, cependant, qui se situent à l'intérieur de l'une ou l'autre sphère, ne remettent pas en cause le principe de la sélection du thème présuffixal ${ }^{1}$, savant ou populaire. On se propose de montrer que cette sélection ellemême entre dans le jeu des contraintes et peut ou non être satisfaite suivant qu'elle converge avec les autres contraintes ou bien se heurte, ponctuellement, à plus forte qu'elle. On verra d'abord, cependant, que les choses ne se présentent pas de la même façon suivant qu'il s'agit d'un verbe, d'un nom ou d'un adjectif, parce que l'articulation entre flexion et dérivation dans les espaces thématiques ne se fait pas de la même façon pour les trois catégories.
\end{abstract}

\section{Flexion et dérivation dans les espaces thématiques}

On ne reprendra pas ici, faute de place, une présentation de la notion d'espace thématique, que l'on supposera connue. Rappelons simplement un point : dans le lexique hérité, les différents thèmes qui constituent l'espace thématique d'un lexème sont inscrits dans le lexique, enregistrés dans la mémoire lexicale des locuteurs. Mais ils n'existent que dans la mesure où ils sont manifestés dans la flexion du lexème ou dans des dérivés existants. La phonétique historique peut expliquer pourquoi le thème $\mathrm{B}^{2}$ de FRAIS est /fref/, mais en synchronie il a cette forme simplement parce que le féminin de cet adjectif est fraîche et que ses dérivés sont fraîcheur ou fraîchir. Dans le lexique en formation, la question qui se pose est par conséquent : comment va se constituer l'espace thématique des nouveaux venus?

Dans les verbes, d'autre part, la présence de la flexion est massive. Sauf pour quelques verbes défectifs, toutes les cases de l'espace thématique sont obligatoirement renseignées. Dans les néologismes, tous les thèmes sont automatiquement productibles : soit par défaut et identiques entre eux, soit conformément à une alternance identique pour tous (thèmes 9 et $12^{3}$ en /e/, thème 13 en /at/). Seules font exception les formations inchoatives en -ir, mais elles suivent toutes le même moule (thème 1 en /is/, thème $11 \mathrm{en} / \mathrm{i} /{ }^{4}$ ). Le thème le plus utilisé par la dérivation est fortement présent dans la flexion : atterrissage sera construit comme nous atterrissons, il atterrissait. Dans les noms, en revanche, la flexion ne joue qu'un rôle secondaire. Seule est présente la flexion en nombre, mais elle est marquée le plus souvent en dehors du lexème lui-même et le thème de pluriel (qui n'est différent du thème A que pour quelques noms en -al et les encore plus rares EUUF, BEEUF et (EIL) n'intervient pas dans la dérivation. La Forme de Liaison Masculin 
Singulier (FLMS) ${ }^{5}$, rarement utilisée, n'interfère pas non plus. Les thèmes $B$ et $S$, sélectionnés par la dérivation, sont extérieurs à la flexion. Et leur formation n'est pas automatique : ni identiques par défaut au thème $\mathrm{A}$, ni déduits par des règles phonologiques. Dans les néologismes, le thème $\mathrm{B}$ n'est identique (en général) au thème $\mathrm{A}$ que si celui-ci a une finale consonantique non suivie dans la graphie d'une consonne muette. Dans les autres cas, il est phonologiquement imprévisible (infra, §5). Pour le thème $\mathrm{S}$, les règles de postériorisation des voyelles moyennes, d'assibilation ou d'épenthèse (Dell \& Selkirk, 1978) ne concernent qu'une partie des lexèmes. Si un nom n'a pas de dérivés - parce qu'il vient d'entrer dans la langue, parce qu'il n'en jamais eu, parce qu'ils sont obsolètes ou démotivés -, son espace thématique est incomplet : il n'a pas de thème $\mathrm{B}$ ni de thème $\mathrm{S}$. Quel est le thème $\mathrm{B}$ de EAU ? /ev/ à cause d'évier, démotivé ? Les adjectifs occupent, vis-à-vis de la flexion, une place intermédiaire. Ils sont concernés plus souvent par la FLMS et la flexion en nombre (les dérivés en -al y sont une classe ouverte), mais elles n'interfèrent pas pour autant avec la dérivation. La flexion en genre, en revanche, est toujours présente. Ils ont donc obligatoirement un thème $\mathrm{B}$, sélectionné également pour la suffixation populaire. Mais un grand nombre d'adjectifs, dits « invariables », ont pour la flexion un thème B identique par défaut au thème $\mathrm{A}$ (une robe marron), ce qui ne les empêche pas d'avoir pour la dérivation un thème $\mathrm{B}$ alternant (marron(n)âtre).

La conséquence de cet état des choses est que l'articulation entre flexion et dérivation dans chaque catégorie, l'influence de l'une sur l'autre, n'est pas la même : forte pour les verbes, faible pour les adjectifs, nulle pour les noms. Dans les verbes, les cas de «surabondance» sont rares. Rarissimes pour une forme fléchie ( (je) puis / (je) peux, (je, il) balaye / (je, il) balaie), peu nombreux pour deux formes présuffixales ou une forme fléchie et une forme présuffixale ou dérivée (nettoiement / nettoyage, endormons / endormissement, tondu / tonte). Dans les noms et les adjectifs, elles sont très fréquentes. Non seulement pour les nouveaux venus (le dérivé en -ien construit sur OBAMA pourra être obamien, obamaien, obamanien, obamatien, obamasien, obamalien...), mais aussi pour le lexique hérité (sur CHAPEAU, l'existence de chapelier n'a pas empêché la formation de chapeauter). On peut penser que dans un cas la prégnance de la flexion entraîne une grande stabilité, et que dans l'autre son évanescence permet beaucoup plus de liberté. Pour la même raison, on observe pour les verbes peu de discordances entre le thème tel qu'il figure dans l'espace thématique et le radical effectivement présent dans le dérivé, tandis que pour les noms ils sont nombreux : troncations (BOUTEILLE $\rightarrow$ boutanche), insertion d'un interfixe (GOUTTE $\rightarrow$ gouttelette), substitutions diverses, par ajout (ROI $\rightarrow$ royaliste) ou par effacement (SACRISTIE $\rightarrow$ sacristain).

La réflexion sur les espaces thématiques est partie de la flexion verbale, et chez certains de ses acteurs n'a porté que sur la flexion verbale (ou n'a été étendue qu'à l'adjectif, à propos de sa flexion). D'où l'impression que l'on pouvait extrapoler à la dérivation ce que l'on observait dans la flexion. Il faut, en fait, inverser la perspective. L'espace thématique d'un lexème n'est pas un donné intangible, consubstantiel au lexème dès sa naissance. Il est, certes, attaché au lexème, il fait partie de son existence lexicale, mais il est à construire et sa conservation, le respect des formes qui y sont déjà inscrites, ne sont que l'effet d'une contrainte parmi d'autres. On le constate pour la flexion (la forme normée avare n'empêche pas que pour beaucoup de locuteurs le féminin d'AVARE soit avarde), y compris pour le verbe (les espaces thématiques actuels sont le résultat d'un nombre considérable de remaniements au cours de l'histoire, et la « grammaire des fautes » montre que pour beaucoup de locuteurs ces remaniements ne sont pas terminés). A fortiori pour le thème présuffixal d'un nom ou d'un adjectif. Faute de place, nos observations ne porteront ici que sur ces deux catégories, dont l'espace thématique a été moins exploré, en attendant de les étendre à celui des verbes.

\section{$2 \quad$ L'inscription dans le lexique : les séries dérivationnelles}

Un mot construit est construit pour entrer dans le lexique (ne serait-ce que momentanément, dans le cas des occasionnalismes) et se trouve obligatoirement au croisement entre un paradigme - la série dérivationnelle : ensemble des mots construits selon un même procédé - et un réseau - la famille dérivationnelle : ensemble des mots construits, directement ou indirectement, sur une même base. Il est naturel, par 
conséquent, que l'insertion dans ces deux ensembles joue un rôle déterminant dans la forme qui lui sera donnée. Commençons par le premier.

\subsection{Analogie à gauche, analogie à droite}

Le mécanisme de l'analogie qui est à l'origine du choix du suffixe (Aronoff, 2007 ; Booij, 2010) détermine également la forme du radical. C'est parce qu'il y a déjà châtaignier et châtaigneraie, palmier et palmeraie, bananier et bananeraie, etc., que -aie sera choisi pour nommer une plantation de manguiers. Pour la même raison, la finale /je/ de MANGUIER deviendra /or/ dans mangueraie.

$\begin{array}{ll}\text { PALMIER } & \text { /je/palmier } \sim / \mathrm{or} / \text { palmeraie } \\ \text { BANANIER } & \text { /je/ bananier } \sim / \mathrm{or} / \text { bananeraie } \\ \text { MANGUIER } & \text { /je/ manguier } \sim / \mathrm{or} / \text { mangueraie }\end{array}$

C'est ce processus analogique qui commande le choix entre le thème " populaire » et le thème « savant », sur des bases similaires, en fonction du suffixe : -eux /ø/ deviendra /øz/devant -eté et /oz/ devant -ité parce que les choses se passent comme cela dans le lexique hérité.
CURIEUX
/ø/ curieux $\sim / \varnothing \mathrm{z} /-\sim / \mathrm{oz} /$ curiosité
GRACIEUX
/ø/ gracieux $\sim / \varnothing \mathrm{z} /$ gracieuseté $\sim / \mathrm{oz} /$ -
CLASSIEUX
/ø/ classieux $\sim / \varnothing \mathrm{z} /$ classieuseté $\sim / \mathrm{oz} /$ classiosité $^{6}$

Le mécanisme de l'analogie est donc orienté à la fois à gauche (similarité des finales dans la forme libre du lexème base) et à droite (même suffixe). Dans d'autres séries dérivationnelles, devant d'autres suffixes, -ier/je/ devient/jer/ et non /ər/ : daumiéresque, aciéreux, pompiérisme, courriériste... Et l'identité phonologique à gauche, devant un suffixe donné, ne suffit pas. L'alternance /عl/ /al/, systématique devant -isme et -iste pour les adjectifs en -el / -elle (qui sont tous des dérivés suffixaux), ne fonctionne pas pour parallèle (qui n'en est pas un) et pour les bases nominales : formalisme, matérialisme, naturalisme, paternalisme, spiritualisme... d'un côté, parallélisme, machiavélisme, blondélisme, pastelliste, nouvelliste... de l'autre.

Ces alternances, par conséquent, n'obéissent pas à un conditionnement purement phonologique. Dans mangueraie, l'apophonie /e/ /ə/, provoquée jadis par le déplacement de l'accent, n'est plus à l'œuvre depuis des siècles. Dans formalisme, il n'y a pas de raison qu'un / $/$ / devienne /a/ parce qu'on introduit un /i/ dans la syllabe qui suit. Elles ne sont pas, non plus, uniquement morphophonologiques, sinon un même suffixe déclencherait toujours la même alternance dans un même contexte phonologique à gauche. On voit avec les bases en / $/ \mathrm{l} /$ que l'appartenance de la base à une série dérivationnelle est déterminante. Il s'agit donc, en fait, d'un phénomène lexical (ou, plus précisément, la traduction morphophonologique d'un phénomène lexical) : un jeu de correspondances entre deux séries dérivationnelles.

\subsection{Analogie par procuration}

Le mécanisme fonctionne à plein, comme dans les exemples ci-dessus, quand la base appartient à une série dérivationnelle : le suffixe confère au lexème son espace thématique. Sans contexte à droite, dans la flexion, un adjectif en -eux aura automatiquement un féminin en -euse, un dérivé en -ard automatiquement un féminin en -arde. On peut néanmoins le voir à l'œuvre dans le cas contraire, quand le lexème n'appartient pas à une série dérivationnelle. Soit, par exemple, BANLIEUE. Il n'a pas de dérivés antérieurs, donc pas de thème $\mathrm{B}$ hérité (apparenté historiquement à LIEUE, dont il est un composé, il a complètement rompu le lien sémantique et, de toute façon, LIEUE non plus n'a pas de thème B). Pour désigner les habitants de la banlieue avec un dérivé en -ard, un thème $\mathrm{B}$ identique au thème $\mathrm{A}$, qui donnerait ${ }^{\circ}$ banlieuard, n'est pas impossible, mais la contrainte de syllabation requiert une accroche consonantique pour le suffixe et il n'y a pas dans le lexique de mots en -euard(e) qui serviraient de modèle. Une épenthèse en /t/, la plus fréquente, donnerait ${ }^{\circ}$ banlieutard. Mais l'alternance $/ \varnothing / \sim / \varnothing t /$ est rarissime en français (introduite seule- 
ment pour former quelques verbes comme bleuter ou équeuter). En revanche, les alternances $/ \varnothing / \sim / \varnothing z /$ sont très fréquentes à cause des adjectifs en -eux / -euse. Face à une difficulté semblable, elles ont déjà inspiré bondieuserie et bondieusard (et plus tard bourdieusisme et depardieuserie). C'est donc banlieusard qui sera adopté. Plus récemment, pour désigner la transformation d'une zone rurale en banlieue de la ville voisine, la langue a eu besoin d'une forme en -isation. Le suffixe -iser appelle normalement un thème savant, mais BANLIEUE n'a pas d'étymon latin disponible et la postériorisation qui fonctionne pour -eux -os- ne s'étend pas à -eue. A défaut, on disposait cette fois du thème utilisé pour banlieusard, mais ${ }^{\circ}$ banlieusiser, avec ses deux /z/ trop rapprochés, se heurte à la contrainte dissimilative. Sera donc sollicitée une autre accroche relativement fréquente après -eu-, à cause des nombreuses bases en -eur:/r/. D'où banlieuriser, qui voisinera avec monseigneuriser, pasteuriser, conteneuriser. Conclusion: quand un lexème n'appartient pas à une série dérivationnelle, il se raccroche à la série la plus voisine.

\subsection{Effet boule de neige et effet de manque}

Cet exemple illustre la face la plus visible, positive, du mécanisme de l'analogie : l'effet boule de neige (le semblable attire le semblable). Mais aussi, moins évidente, la face négative : si le semblable n'existe pas, s'il n'y a pas (ou très peu) de précédents dans le lexique, la langue cherchera autre chose. Un ancien président de la République a parlé à plusieurs reprises de " mécanisme péren », « dispositif péren », «financements pérens ${ }^{7}$ : un masculin en $/ \varepsilon n /$, a fortiori s'il est écrit -enne comme PERENNE et non -ène comme EPICENE, est exceptionnel en français. Il est donc spontanément refait sur les masculins des adjectifs alternants en -EIN/-EINE, -AIN/-AINE ou -IEN/-IENNE. Parmi les lexèmes en /ar/, on a observé depuis longtemps que les adjectifs épicènes (AVARE, BIZARRE) tendaient à s'aligner sur les adjectifs alternants en -ard/-arde, beaucoup plus nombreux, et la Toile montre aujourd'hui que les formes comme avarde ou bizarde continuent à être abondamment attestées. Pour la dérivation, les noms en -ar n'ont pas besoin d'épenthèse consonantique, mais pour la même raison BAZAR donnera bazarder comme HASARD donne hasarder. L'attraction de l'alternance /ar/ /ard/ s'étend jusqu'aux finales en -oir : renoirdesque a été entendu dans une émission culturelle de France Inter et peut être relevé sur la Toile, où l'on trouve également (entre autres) noirdesque, pied-noirdesque, trou-noirdesque, point noirdesque et Chat noirdesque.

Inversement, pour construire un dérivé en -at sur un nom en -ard, en l'absence de thème savant spécifique, on s'attendrait logiquement à ce que soit retenu le thème en /ard/ disponible. Or on constate sur la Toile que le statut de SMICARD, ou la collectivité des smicards, est le smicariat, ceux des CONNARDs le connariat et ceux des MOTARDs le motariat. Simplement parce qu'il n'y avait pas, semble-t-il, jusqu'à une époque récente, de dérivés en -at construits sur de telles bases, donc pas de dérivés en -ardat, tandis que les dérivés en -ariat (notariat, secrétariat, volontariat...) se comptent par dizaines à cause, au départ, des nombreuses bases en -aire. Il n'y avait pas, non plus, jusqu'au milieu du XXe siècle, de dérivés en -at sur des bases en -ant (qui ne posent pas problème pour la dérivation savante puisqu'elles n'ont pas besoin d'être latinisées). Le statut d'ASSISTANT a donc été l'assistanat, qui faisait écho au décanat, au sultanat et à l'artisanat, plutôt que l'assistantat (qui se répand néanmoins aujourd'hui à cause du sens péjoratif qu'a pris assistanat dans d'autres contextes). Dans les conversions $\mathrm{N} \rightarrow \mathrm{V}$, les bases en /i/, /y/, /e/ sans consonne finale graphique peuvent donner des dérivés en -ier (skier) -uer (charruer), -éer (agréer) parce qu'il y a déjà, dans le lexique hérité, des verbes dotés d'une finale semblable (nier, suer, créer) tandis que pour les bases en $/ \varnothing /, / 0 /, / \mathrm{a} /$ l'épenthèse (ou la troncation) s'impose (équeuter, pianoter, blablater) parce qu'il n'y a pas de verbes en ${ }^{*}$-euer, ${ }^{*}$-oer, ${ }^{*}$-aer ${ }^{8}$. De rarissimes exceptions (des cas particuliers, en fait) permettent de préciser où est le problème. On trouve sur la Toile des attestations de brouhaher, cahincaher, canoer 'faire du canoë', où la finale du radical résulte d'une troncation ou d'une haplologie. Mais ces verbes ne semblent pas pouvoir être conjugués aux personnes où le radical est à nu : ? je cahincahe, ?? il(s) canoe(nt). Une finale vocalique est possible, à ces personnes, dans la conjugaison en -er, avec d'autres voyelles (il skie, il sue, il crée). Elle est possible, avec ces voyelles, dans d'autres conjugaisons (il bat, il vaut). Elle n'est pas possible pour ces voyelles dans la conjugaison en -er parce qu'il n'y a pas de précédents. 


\subsection{Effet boule de neige et effet de rime}

L'effet boule de neige est aussi un effet de rime, mis en évidence par Roché (2007) dans la dérivation en -isme mais qui touche toutes les séries dérivationnelles. Le suffixe constitue le plus souvent ce qu'en poésie on appelle une rime «suffisante» (voyelle + consonne), parfois une rime «pauvre» (voyelle seule). Or, pour marquer l'appartenance du mot construit à la série dérivationnelle dans laquelle il doit s'intégrer, une rime « riche » (qui remonte au-delà de la dernière voyelle) est préférable à une rime pauvre ou suffisante. Pour cela, plusieurs processus conduisent au même résultat. Le suffixe peut être remplacé par une variante plus longue : à force d'être ajouté à des bases en -ier dont la finale devient /ər/ (CHEVALIER $\rightarrow$ chevalerie), le suffixe -ie est devenu -erie, d'où GENDARME $\rightarrow$ gendarmerie à côté de MAIRE $\rightarrow$ mairie. Une base nominale peut être remplacée par l'adjectif de relation correspondant, comme dans les dérivés en -alisme cités plus haut (le formalisme, le spiritualisme, le matérialisme sont fondés sur le primat donné à la forme, à l'esprit, à la matière). Et le thème attendu peut être remplacé par un thème irrégulier : smicariat rime richement avec des dizaines de dérivés en -at, tandis que dans ${ }^{\circ}$ smicardat la rime serait réduite à une assonance. Le suffixe -itude constitue en lui-même une rime riche, mais il se trouve en plus que dans les dérivés hérités du latin (aptitude, béatitude, certitude...) les radicaux en /t/ sont surreprésentés. De sorte que /t/ sera l'épenthèse privilégiée devant ce suffixe (gagatitude, neuneutitude, blaireautitude...), alors que la contrainte dissimilative (infra, § 6) devrait l'interdire (Plénat, 2011 : 165). On voit même apparaître une variante -titude après des bases qui n'ont pas besoin d'épenthèse (mectitude, plouctitude, cheaptitude...). Les divers processus qui tendent à enrichir la rime se confondent et s'interpénètrent. Peu importe, dans tempétueux, que -ueux soit une variante de -eux ou bien tempétu- un thème irrégulier calqué sur ceux de la quatrième déclinaison latine (cf. INCESTE $\rightarrow$ incestueux); dans robusticité que -icité soit une variante de -ité et dans pathogénicité que PATHOGENE soit remplacé par pathogénique (qui a un autre sens) : l'essentiel est que, parmi les dérivés en -eux, les sous-séries en -ueux ou en -ieux s'étendent aux dépens des finales moins marquées, parmi les dérivés en -ité les sous-séries en -icité et -alité, parmi ceux en -isme les sous-séries en -alisme et -ianisme, etc.

\section{5 Écarts, régularités, contrainte}

L'analogie, l'effet boule de neige entraînent toutes sortes d'écarts. Une alternance rare tend à être remplacée par une alternance plus fréquente. Sur SIROP, on trouve dans les dictionnaires siroper et siropier, mais seul siroter est usuel, en dépit de la graphie du primitif. Sur TABAC, seul le versant savant a conservé des alternances en vélaire ou palatale (tabacomanie, tabagie), la dérivation populaire les a remplacées par /t/ (tabatière). FER BLANC a conservé la graphie de son formant de droite, mais pas l'allomorphie qui le caractérisait (ferblantier). D'une façon générale, /t/, déjà très présent comme consonne latente, s'étend aux dépens des vélaires et des palatales, particulièrement instables, des labiales, encore plus rares dans ce rôle, ou de /1/ (cf. CUCUL $\rightarrow$ cucuterie). Sauf raison particulière, c'est /t/ qui se généralise pour fournir une épenthèse dans la dérivation verbale. Mais dans une sous-série bien délimitée, celles des verbes construits sur le cri d'un animal, c'est /l/ qu'on trouvera le plus souvent quand l'onomatopée correspondante ne se termine pas par une consonne : bêler, boubouler, caracouler, chouler, coucouler, crouler, feuler, hôler, miauler, pupuler... Sans doute à cause des formations latines où /1/ était apporté par le suffixe -ulare (bubuler, striduler, ululer, zinzinuler...). C'est /1/ encore qui fournit l'épenthèse dans congolais et togolais, à cause du voisinage géographique avec sénégalais - autre illustration du rôle des sous-séries référentielles, donc du paramètre lexical.

Il ne faudrait pas, cependant, que des exemples plus ou moins pittoresques qui mettent en évidence les effets de l'analogie fassent oublier l'essentiel. L'analogie n'est pas quelque chose de marginal qui s'opposerait aux règles. Au contraire : c'est elle qui commande les régularités, comme on l'a montré au début de cette section. Et elle oblige à s'interroger sur ce qui est perçu comme «normal ». Pourquoi $b a-$ zarder attire-t-il l'attention et pas hasarder? boulevaresque et pas boulevardier? Parce que la perception de ce qui est régulier et de ce qui ne l'est pas est fondée sur la graphie (infra, § 5). Phonologiquement, c'est hasarer et boulevaresque (attestés l'un et l'autre) qui sont réguliers, puisqu'ils éliminent 
l'allomorphie. Il y a une régularité phonologique, qui justifie ces deux formes. Une régularité phonographique, qui justifie hasarder et boulevardier. Et une régularité lexicale, qui justifie hasarder, bazarder et boulevardier.

Pour exprimer cette régularité sous forme de contrainte, puisqu'elle peut s'opposer à d'autres, on l'énoncera ainsi :

Contrainte de série : le thème sélectionné est la forme qui, par rapport à la forme libre, correspond dans le lexique aux alternances les plus fréquentes dans le même contexte à droite.

Quand le lexème appartient à une série dérivationnelle, la contrainte assure la correspondance entre cette série et celle du dérivé. Dans le cas contraire, elle tend à intégrer le lexème à une série dérivationnelle.

\section{$3 \quad$ L'inscription dans le lexique : les familles dérivationnelles}

Un internaute forge plombesque pour qualifier un joueur qui, d'après lui, mérite le prix du Ballon de plomb de l'année. Il n'a évidemment pas réfléchi à la forme qu'il allait donner à ce mot et elle n'étonne pas le lecteur. Le morphologue dira qu'elle était attendue, régulière, puisque -esque requiert le thème $\mathrm{B}$ et que le thème B de PLOMB est /plãb/. Mais, on l'a déjà fait remarquer, le thème B de PLOMB n'est /plãb/, en synchronie, que du fait de sa présence dans plomber et plombier. Dire « PLOMB donne plombesque parce que le thème B de PLOMB est /plõb/ » revient à dire « PLOMB donne plombesque parce que le thème /plã b/ est déjà utilisé dans des dérivés antérieurs, présent dans la famille dérivationnelle du lexème ».

Autre exemple : notre internaute, ou un autre, s'intéresse au genou d'un joueur. Il parlera de ses ennuis genouesques (168 «résultats» via Google, 06.11.2013) plutôt que genouillesques (42 résultats). Le thème $\mathrm{B} / 3 ə n u j /$ peut donc être utilisé, mais dans ce cas il ne s'impose pas. La formule «GENOU donne genouillesque parce que le thème /zənuj/ est déjà utilisé dans des dérivés antérieurs 》 reste vraie (un thème en /uj/ n'aurait aucune chance de se manifester s'il n'était pas déjà présent dans genouillère et dans s'agenouiller) mais elle doit être fortement modalisée en «GENOU peut donner genouillesque... », ou « GENOU donne normalement genouillesque... ». La raison de la différence de traitement, entre ces deux exemples, saute aux yeux : pour PLOMB, l'allomorphie en $/ \mathrm{b} /$ est soutenue par la graphie tandis que pour GENOU la graphie de la forme libre n'enregistre pas la consonne latente.

Le principe d'unicité du thème («le thème $\mathrm{B}$ de PLOMB est /plãb/ ») n'est donc en fait qu'une contrainte, une contrainte forte, mais qui a besoin d'être soutenue par d'autres (en l'occurrence : la graphie) et qui peut s'opposer à d'autres, comme on l'a vu avec banlieuriser, qui ne reprend pas le thème utilisé pour banlieusard. Une contrainte qui peut être formulée :

Contrainte de famille : le thème sélectionné est une forme déjà attestée dans la flexion ou dans la famille dérivationnelle du lexème.

C'est, comme la précédente, une contrainte d'ordre lexical et les deux sont complémentaires, puisqu'elles reposent sur les deux axes fondamentaux de la construction des lexèmes. Dans nos deux exemples, la contrainte de série est inopérante. Pour PLOMB, sur une base purement phonologique à partir de la forme libre /plõ/, elle donnerait un dérivé en -on(n)esque qui rejoindrait une abondante sous-série. Mais elle est bloquée par la graphie. Pour GENOU, elle est inopérante parce que les finales en /u/ sont peu nombreuses en français et se partagent entre diverses alternances dont aucune n'est suffisamment représentée pour être attractive : /u/ / $\mathrm{l} / /$ (MOU), /u/ /us/ (DOUX, ROUX), /u/ /uz/ (EPOUX), /u/ /ut/ (EGOUT), /u/ /up/ (COUP), $/ \mathrm{u} / \sim / \mathrm{uv} /$ (LOUP), $/ \mathrm{u} / \sim / \mathrm{uj} /$ (POU), $/ \mathrm{u} / \sim / \mathrm{ul} /$ (BAGOU), $/ \mathrm{u} / \sim / \mathrm{un} /$ (TOUTOU). De ce fait, s'il n'obéit pas à la contrainte de famille, le locuteur se rabat sur la forme libre et produit genouesque (infra, § 5). Il peut aussi produire genoutesque, lui aussi bien attesté : rare après /u/, l'alternance en /t/ est suffisamment fréquente d'une façon générale pour représenter une solution de rechange. Il produira aussi genouter s'il a besoin d'un verbe autre qu'agenouiller (pour 'donner un coup de genou', par exemple) : c'est dans la dérivation verbale, on l'a dit, que l'épenthèse en / $t / \mathrm{s}$ 'impose avec le plus de constance. 
Le poids de la contrainte de famille est très variable et difficile à isoler dans la mesure où elle coïncide le plus souvent avec les autres contraintes. On peut observer cependant qu'il dépend à la fois de raisons internes et du poids plus ou moins grand des contraintes concurrentes. Il est nul, en toute logique, quand il n'y a ni flexion ni dérivés antérieurs, comme on l'a vu pour BANLIEUE. Les premiers dérivés de CAFE, quand le mot est apparu, ont pu être cafier, caféier, cafetier. Il est faible quand les seuls dérivés existants sont peu employés ou obsolètes, surtout si l'alternance qui s'y manifeste n'est pas soutenue par des séries lexicales abondantes, comme c'était le cas pour SIROP ou TABAC. Pour CHOU, l'allomorphie historique en /1/ qui se manifeste dans choulière est abandonnée pour /t/ quand il s'agit du légume (choutière) ou d'un terme d'affection (ma choute), pour /p/ quand il s'agit d'une coiffure (choupette), pour $/ \mathrm{k} /$ en pâtisserie (chouquette). Il est fort, en revanche, quand la flexion s'ajoute à plusieurs dérivés antérieurs : malgré la graphie, FRAIS donne fraîchitude. On trouvera dans les sections suivantes des exemples où la satisfaction d'une autre contrainte entraîne une violation de la contrainte de famille. On laissera de côté, en revanche, les cas où le polymorphisme résulte d'aléas de l'histoire phonétique (VERT verte / verdure, LONG longer / longueur). Ils sont relativement nombreux et battent en brèche le principe d'unicité pour une case de l'espace thématique donnée.

\section{$4 \quad$ L'inscription dans le lexique : les deux registres}

Depuis la relatinisation qui s'est opérée en moyen français (et poursuivie dans les périodes suivantes), le lexique français se déploie sur deux versants : «populaire» et « savant ». Rappelons très sommairement les données. Au lexique d'origine latine ou germanique, transmis par voie orale et profondément modifié par les évolutions phonétiques, sont venus s'ajouter un grand nombre d'emprunts, intégrés non pas tels quels mais simplement adaptés au français. Sur le modèle de ces emprunts, des mots nouveaux encore plus nombreux ont été créés, soit sur des bases empruntées pour cela au latin, soit sur des bases déjà présentes en français. Il en est résulté, pour la morphologie constructionnelle, un système dual, avec ses affixes dédoublés : -ité vs -eté, -aire vs -ier, -at vs -é, -eur/-rice vs -eur/-euse, etc.; ses thèmes spécialisés : EVEQUE épiscop-at vs évéch-é, GLACER glaciat-ion vs glaç-age; des modèles différents pour la composition néo-classique et pour la composition traditionnelle; etc. Tout cela est bien connu dans ses grandes lignes mais apparaît beaucoup plus complexe quand on y regarde d'un peu près. Il n'est pas possible d'entrer ici dans le détail, on ne pourra qu'esquisser une réponse aux deux questions principales pour la morphophonologie : comment est formé le thème savant ? comment se fait le choix entre thème savant et thème populaire?

\subsection{Le thème savant}

D'une façon générale, et en schématisant quelque peu ${ }^{9}$, on constate que les thèmes présuffixaux savants sont de deux sortes :

(1) Dans une série dérivationnelle donnée, une même alternance $A \sim S$ s'impose à tous les lexèmes et n'affecte que la dernière rime. Elle peut être identique à l'alternance $\mathrm{A} \sim \mathrm{B}(/ \mathrm{if} / \sim / \mathrm{iv} / \mathrm{dans}$ ACTIF $\rightarrow$ activité comme dans le féminin active ou dans OISIF $\rightarrow$ oisiveté) ou bien spécifique et se distinguer soit d'un thème B non alternant $(/ \varepsilon \mathrm{l} / \sim / \mathrm{a} 1 /$ dans FORMEL $\rightarrow$ formalité $\mathrm{vs} / \varepsilon \mathrm{l} / \sim / \varepsilon \mathrm{\varepsilon l}$ dans formelle), soit d'un thème B qui alterne autrement $(/ \varnothing / \sim / \mathrm{oz} /$ dans CURIEUX $\rightarrow$ curiosité vs $/ \varnothing / \sim / \varnothing z /$ dans curieuse $)$. Le plus souvent, si l'on part du thème $\mathrm{B}$, la formation du thème savant se traduit par le déplacement d'une voyelle moyenne vers l'arrière $(/ \varepsilon />/ \mathrm{a} /, / Ø />/ \mathrm{O} /)$ ou par une épenthèse vocalique $(-b l e>b i l)$, ce qui a conduit à formuler des règles $a d$ hoc. Mais elles ne s'appliquent que dans les limites de ces séries. Par définition, puisqu'il s'agit de séries dérivationnelles, les lexèmes concernés sont en principe des lexèmes construits (mais lesdites séries peuvent agréger, par analogie, des lexèmes qui ne le sont pas) et l'alternance qui les caractérise concerne aussi bien les lexèmes formés en français que ceux qui ont été empruntés au latin: TELECHARGEABLE fait téléchargeabilité comme INSATIABLE (lat. INSATIABILIS) fait insatiabilité. 
(2) Le lexème n'appartient pas à une série dérivationnelle. Le thème $S$ peut être identique au thème $\mathrm{A}$ (et dans ce cas identique également au thème $\mathrm{B}$, en général) : CONSUL $\rightarrow$ consulat. Ou bien, plus souvent, idiosyncrasique et emprunté au latin, soit directement pour construire le dérivé (EIL $\rightarrow$ oculiste), soit indirectement si celui-ci a été formé en latin (EVEQUE $\rightarrow$ épiscopat). Ou encore forgé pour imiter une forme latine (BOURG-LA-REINE $\rightarrow$ réginaburgien). Les variations, comme on le voit sur ces exemples, peuvent affecter n'importe quelle partie du mot et ajouter une ou plusieurs syllabes. Contrairement aux thèmes $\mathrm{S}$ de la catégorie précédente, ceux-ci ne peuvent se rencontrer que si le lexème concerné a un étymon latin, authentique ou controuvé.

Les deux types de thèmes peuvent se rencontrer pour un même lexème, y compris devant un même suffixe : GOUVERNEUR peut donner (entre autres) gouvernorat ou gubernatorat, RIGOUREUX rigourosité ou rigorosité. Ils ne procèdent pas de la même démarche. Les premiers relèvent de l'allomorphie, comme les alternances entre la forme libre et le thème présuffixal populaire. Ils n'altèrent pas davantage le lexème, et ils ne l'altèrent qu'en fonction de son environnement. Les seconds relèvent du supplétisme : ils remplacent le lexème par une forme empruntée à une autre langue (que cette langue soit historiquement l'ancêtre du français ne change rien au fond). Il n'y a pas de solution de continuité entre ces substitutions, qui consistent à remplacer la forme moderne du lexème par son étymon latin, et celles qui la remplacent par un thème emprunté à un autre mot de la même famille (patern- pour PERE dans paternel), par un mot latin non apparenté (fong- pour CHAMPIGNON dans fongique) ou par un équivalent grec (ot- pour OREILLE dans otite). Pour EAU, le thème latin /akw/ est phonologiquement aussi éloigné de /o/ que le thème grec /idr/.

\subsection{Quelle(s) contrainte(s) ?}

Ce qu'il y a de commun aux deux types de thèmes, c'est qu'ils permettent de «faire savant ». Pour satisfaire une logique qui, ici encore, est d'ordre lexical. Puisque dans le lexique hérité les dérivés en -at, par exemple, sont précédés d'un thème savant, les dérivés nouveaux le seront aussi. On peut « faire savant» soit en latinisant la finale du radical (et gouverneur deviendra gouvernor-, rigoureux deviendra rigouros-), soit en remplaçant le thème populaire par un thème emprunté à l'étymon latin (et gouverneur deviendra gubernator-, exil deviendra ocul-). Ou encore en lui substituant un équivalent grec ou latin (et oreille deviendra $o t-$ ). La contrainte porte donc sur la correspondance entre thème et mode de formation, et peut se formuler :

Contrainte de registre : le registre (populaire ou savant) du thème sélectionné doit être identique à celui du mode de formation.

Mais elle ne peut fonctionner qu'en association avec l'une ou l'autre des deux autres contraintes lexicales. Soit la contrainte de série, et l'accord avec le mode de formation se traduira par une simple latinisation de la finale. Soit la contrainte de famille, qui dans ce cas mobilisera un membre plus éloigné, l'ancêtre latin, ou un parent par adoption. Gouvernor-at dans un cas, ocul-iste ou ot-ite dans l'autre.

Dans le premier cas, en fait, la contrainte de série serait suffisante pour produire les formes souhaitées, puisqu'elles procèdent d'une relation de correspondance entre deux séries dérivationnelles. Il y a déjà DOCTEUR et doctorat, RECTEUR et rectorat, PROFESSEUR et professorat, etc., ANIMATEUR fera donc animatorat. C'est la raison pour laquelle ces allomorphies peuvent concerner des lexèmes qui n'ont rien de latin. Pris dans le jeu de l'analogie, les noms d'agent en -er d'origine anglaise donneront des dérivés en -orat (leadorat, supportorat, webmastorat...) s'ils sont prononcés comme les noms en -eur, des dérivés en -ariat (leadariat, supportariat, webmastariat...) s'ils sont prononcés comme les noms en -aire. On voit bien, également, qu'il s'agit d'un simple mécanisme analogique lorsqu'une série attire des éléments phonologiquement proches (PODESTAT $\rightarrow$ podestariat, comme plus haut SMICARD $\rightarrow$ smicariat), ou bien quand deux séries se télescopent. On trouve un grand nombre de formes en -oriat construites sur des bases en -eur, y compris dans des contextes soignés (le TLF signale assessoriat, auditoriat, dictatoriat, rectoriat), formes qui résultent d'un amalgame entre les allomorphies /œr/ $/ \mathrm{or} / \mathrm{et} / \mathrm{er} / \sim / \mathrm{arj} /$. Dans le second cas, en revanche, la contrainte de famille ne suffirait pas : il faut que la contrainte de registre lui 
enjoigne de rechercher non pas un membre proche (le thème B) mais l'ancêtre latin ou un substitut par adoption.

Pour résumer schématiquement le jeu des contraintes autour de ces mêmes exemples :

- gouverneurat, ceilliste, oreillite ne satisfont pas la contrainte de registre mais sont marginalement attestés parce qu'ils conservent intégralement la forme libre (infra, § 5);

- gouvernorat satisfait la contrainte de registre et la contrainte de série, mais pas la contrainte de famille (gouvernor- n'est pas un thème latin);

- oculiste satisfait la contrainte de registre et la contrainte de famille mais pas la contrainte de série (il n'y pas de correspondance régulière entre oxil et ocul-). Otite également, d'une autre façon ;

- gubernatorat satisfait les trois et devrait être préféré. Si gouvernorat est néanmoins la forme plus souvent retenue, c'est qu'il satisfait la contrainte de registre tout en restant plus proche de la forme libre.

Cette configuration complexe est à l'origine des effets paradoxaux, contradictoires, de la contrainte de registre. Les dérivés en -ité de UNIQUE et de RECIPROQUE sont unicité et réciprocité : le choix du thème savant se traduit pour eux par une spirantisation de la vélaire. On constate sur la Toile que MERDIQUE donne aussi, presque toujours, merdicité et LOUFOQUE plus souvent loufocité que loufoquité, donc que la contrainte de série a fonctionné. À plein pour MERDIQUE, qui appartient à une abondante série dérivationnelle, mais également, dans une moindre mesure, pour LOUFOQUE, par analogie (et pour BAROQUE qui donne barocité dans des proportions encore plus importantes). En revanche, INIQUE donne iniquité (et ANTIQUE antiquité, OBLIQUE obliquité) : pour eux, l'effet de série ne fonctionne pas parce que la spirantisation est bloquée par la contrainte de famille, l'étymon latin ayant une finale en -iquus et non en -icus. Même paradoxe dans les dérivés en -ien de LAOS et de COLOSSE. Le premier s'écrit Laotien, comme Béotien, parce que devant -ien, qui appartient au registre savant, /s/ s'écrit généralement -t-, le second s'écrit Colossien parce que sa base est bien Colosse et pas ${ }^{\circ}$ Colotie. LAOS, qui n'est ni latin ni grec, a été «savantisé » par analogie, tandis que pour COLOSSE, qui est grec, l'analogie est bloquée par la fidélité aux origines.

\subsection{Interférences et conflits}

Les deux domaines, populaire et savant, sont loin d'être étanches. On trouve dès l'ancien français nombre de formes « demi-savantes », où le contact avec le latin a empêché certaines évolutions phonétiques. Dans le lexique hérité, des hybrides combinent un thème savant avec un suffixe populaire : c'est dortoir que la langue a conservé, et pas dormoir ou dormitoire qui étaient plus cohérents, régulier et pas 'réglier ou ${ }^{\circ}$ régulaire. Les suffixes -el et -al ont toutes les apparences d'un doublet populaire/savant, d'autant que -el est latinisé en -al devant un suffixe savant (FORMEL $\rightarrow$ formalité), mais lui-même sélectionne le thème savant (criminel, rationnel, temporel...), comme -al. Inversement, plusieurs formations importantes sont communes aux deux registres : la suffixation en -eux (laiteux, noueux, écailleux... d'un côté, musculeux, sirupeux, cartilagineux... de l'autre); les conversions $\mathrm{N}>\mathrm{V}$ (ordonnancer vs circonstancier, bitumer / bituminer, tabler / tabuler ... ${ }^{10}$ ). Dans ce cas, la contrainte de registre est inopérante. Le choix du thème dépend alors, directement, du type de discours dans lequel le dérivé est destiné à être employé, alors qu'en général le choix du registre, populaire ou savant, commande en même temps le choix du mode de formation et celui du thème, qui doivent être en harmonie (d'où la contrainte de registre). Dans la dérivation en -eur elle-même, il est admis que le choix du suffixe (-eur/-rice vs -eur/-euse) commande celui du thème. Mais c'est plus souvent l'inverse qui se produit: pour les non animés masculins, la forme est la même, et pour les animés, forgés en général d'abord au masculin, c'est la forme du dérivé, en -eur ou en -ateur, qui commandera de les féminiser en -euse ou en -atrice.

La majorité des violations de la contrainte de registre viennent d'une discordance entre le mode de formation et l'environnement (lexical, textuel) du lexème concerné. Le thème savant se substitue au thème populaire attendu, par exemple, quand les naturalistes doivent nommer un biotope en utilisant le suffixe -aie, parce qu'ils n'en ont pas d'autre à leur disposition. Un suffixe qui sélectionne, normalement, le 
thème populaire (chênaie, saulaie, châtaigneraie...), alors que le registre dans lequel ils se situent est celui du discours scientifique. D'où abiétaie, bétulaie, lariçaie, suberaie, viburnaie, etc. (Roché, 2011). En sens inverse, plus souvent, le thème populaire tend à remplacer le thème savant quand un suffixe passe de la langue des clercs à celle de tous les jours. Tant que -isme et -iste servent à nommer des doctrines philosophiques ou religieuses et leurs adeptes, ils sont presque toujours précédés du thème savant. Pour des comportements ou des professions, ils s'accommodent souvent d'un thème populaire (amateurisme, voyeurisme, jeunisme, suivisme, arrivisme... ; chaîniste, fleuriste, médailliste, primeuriste, accessoiriste...). DISQUE devient disc- dans (hernie) discale mais reste disqu- dans disquaire. Le suffixe -ité tend à occuper la place que tenait -eté, qui n'est plus que très peu productif, et perd de ce fait son caractère savant. Les dérivés construits ou reconstruits sur le thème populaire prolifèrent sur la Toile, pour doubler un dérivé existant en -(e)té (chastité, clairité, cruellité, hautainité, tendrité...), en -ité (aveuglité, humblité, idoinité, rondité, viergité...) ou construit autrement (chauvité, chinoisité, jeunité, maigrité, sérieusité...). Et les linguistes eux-mêmes ont forgé sourdité.

D'autres violations de la contrainte de registre ont pour origine la satisfaction de contraintes phonologiques, qui seraient contrariées si elle était respectée. Nous les rencontrerons plus loin.

\subsection{Simplifier l'espace thématique?}

Puisque la sélection du thème ne répond pas à une assignation automatique mais à un ensemble de contraintes, et qu'au thème savant comme au thème populaire peuvent correspondre plusieurs formes, on peut se demander s'il est nécessaire de conserver, dans l'espace thématique, des cases distinctes pour l'un et pour l'autre. Il semble que oui. Pour des raisons qui touchent aux modalités de la variation, d'abord : les systèmes d'alternance ou de supplétion n'y sont pas les mêmes. Pour des raisons internes à la dérivation, ensuite : cette distinction commande un fonctionnement différent de la contrainte de famille. Pour des raisons d'articulation avec la flexion, surtout: dans l'espace thématique des verbes et des adjectifs, le thème présuffixal populaire est commun à la dérivation et à la flexion, tandis que le thème présuffixal savant lui est spécifique. L'opposition de ces deux thèmes s'intègre dans une dualité qui dépasse le cadre morphophonologique. Dans la composition, par exemple, la sélection du thème $\mathrm{S}$ pour le premier formant des composés néo-classiques (laryngologie, comme dans laryngal, et pas *larynxologie) et du thème $\mathrm{A}$ pour les composés traditionnels (coton-tige / kotõtiz/ et pas / koton/ comme dans cotonneux) va de pair avec un fonctionnement différent sur le plan sémantique.

\section{$5 \quad$ La fidélité à la forme libre}

Sans le poids de l'histoire et de la norme, tout le monde dirait les chevals et vous disez. Le «besoin d'invariabilité » (Frei, 1929), ou de plus de régularité, se manifeste par une tendance à éliminer l'allomorphie radicale en conservant la forme libre. Si d'autres contraintes ne s'y opposent pas, le thème présuffixal, populaire ou savant, lui est identique : FOOT, IPOD et BRECHT ne peuvent donner que footeux, ipodesque et brechtien. Lorsque le mot n'a pas de dérivés antérieurs, pas d'étymon latin et n'est pas doté d'une finale qui le rattache à une série dérivationnelle, donc que la contrainte de famille et la contrainte de série sont inopérantes, la conservation de la forme libre au thème $\mathrm{B}$ ou au thème $\mathrm{S}$ produit une forme par défaut. Mais on constate qu'avec des finales phonologiquement identiques LACLOS donne laclosien (pro-

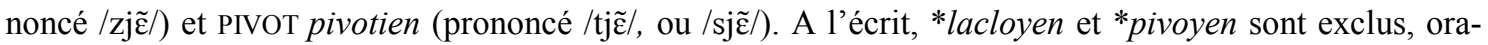
lement /lakloj $\tilde{\varepsilon} /$ et /pivoj $\tilde{\varepsilon} /$ peu vraisemblables. Les conventions orthographiques du français, qui note les consonnes latentes dans la graphie de la forme libre, font qu'en retour une consonne graphique est perçue comme une consonne latente. La conservation de la forme libre - phonique - est entravée par la graphie, qui oriente vers d'autres directions. On constate cependant que les partisans de MONTEBOURG se partagent (outre les montebourgeois) entre montebourgiens et montebouriens : si les premiers tiennent compte de la graphie, les seconds donnent la priorité à la prononciation du patronyme. Certains dérivés sont écrits par des internautes avec, devant le suffixe, un trait d'union (disney-esque, chic-issime, faux-cul-isme...) qui 
semble avoir pour but de protéger la prononciation de la base tout en conservant sa graphie (quand elle est possible, une modification graphique - chiquissime, faux-cuïsme - aboutit au même résultat). Même sans trait d'union, il est vraisemblable que hallidayesque et mickeyesque seraient prononcés /عqsk/ plutôt que

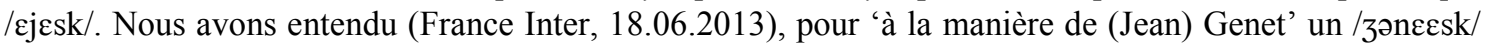
que le locuteur aurait peut-être écrit genetesque.

La forme par défaut du thème $\mathrm{B}$ et du thème $\mathrm{S}$ est commandée, par conséquent, en l'absence de contraintes lexicales ou quand elles sont neutralisées, par une double fidélité à la forme libre : à sa forme phonique et à sa forme graphique. En accord quand la consonne finale graphique est articulée, comme dans les premiers exemples ci-dessus. En conflit quand elle ne l'est pas ou quand elle change de valeur, comme le $-c$ de CHIC qui passe de $/ \mathrm{k} /$ à $/ \mathrm{s} /$ dans chicissime, lui aussi attesté (mais est-il vraiment prononcé ainsi ?). Dans ce cas, il faut ou bien modifier la graphie pour conserver la base phonique (montebourien), ou bien adapter le thème présuffixal à la graphie (montebourgien). À la contrainte de fidélité traditionnelle en phonologie - faithfulness : l'output ne doit rien enlever à l'input, ni rien lui ajouter - il faut ajouter une contrainte visant la correspondance entre la graphie de la base et la forme du dérivé. Soit (le libellé de la première sera commenté plus loin) :

Contrainte de fidélité (phonologique) : le thème sélectionné se rapproche le plus possible de la forme libre.

Contrainte (de fidélité) phonographique : le thème sélectionné est la forme induite par la graphie de la forme libre.

Ensemble ou séparément, elles entrent également en conflit avec les autres contraintes, y compris quand un thème $\mathrm{B}$ ou un thème $\mathrm{S}$ a déjà été conféré au lexème par son insertion dans le lexique. Comme le fait remarquer une internaute, « Pourquoi clou ---> clouer, trou ---> trouer et genou ---> agenouiller plutôt que genouer ou quelque chose d'approchant? » (http://apprendre.cultureforum.net/t1488-agenouiller). Il serait plus naturel, en effet, de dire genouer puisque GENOU s'écrit genou et se prononce /3ənu/. C'est ce que font certains, qui sans doute ignorent s'agenouiller : « et pourquoi pas me genouer et te dire pitié t'es vraiment un spéciale beauté pour moi alors envie de t'inviter au maroc tu seras la bienne venu » (miangemidemon76120.skyrock.com/1816489460). Les contraintes lexicales - en l'occurrence la contrainte de famille - fonctionnent d'autant moins que le lexique du locuteur est pauvre ou mal maîtrisé.

Côté savant, on peut voir les contraintes de fidélité à l'œuvre, par exemple, à propos des spirantisations évoquées plus haut. Les finales en $/ \mathrm{k} /$ qui ne sont pas d'origine gréco-latine et n'appartiennent pas, ou ne sont pas assimilées, à une série dérivationnelle peuvent être spirantisées si elles sont écrites $-c$ dans la forme libre (balzacien, aurignacien, jociste, puciste...) mais ne le sont jamais si elles sont écrites $-k$, $-c k$, - $q$ ou -que (bartokien, kayakiste, bismarckien, gluckiste, péquiste $[\leftarrow P Q=$ Parti Québécois], pétrarquisme, basquiser...). Dans le premier cas la graphie n'a pas besoin d'être modifiée, dans le second elle bloque la spirantisation. Pour les dérivés construits sur des sigles (jociste, jaciste, puciste, tuciste ...), on peut se demander d'ailleurs s'il s'agit vraiment d'une spirantisation, ou plutôt d'une concaténation du suffixe à la forme graphique de la base, et d'une lecture du résultat selon les habitudes phonographiques du français (dans la communication écrite, on reconnaît mieux les rugbymen du PUC s'ils sont appelés pucistes que s’ils étaient les ${ }^{\circ}$ puquistes; oralement, /pykist/ ne semble pas invraisemblable). Dans les formes non spirantisées, minoritaires sur les bases en $-c$, comme chiraquien, kérouaquien ou perecquien, en revanche, c'est la fidélité phonique qui l'a emporté : elle a contrecarré la contrainte de registre et obligé à modifier la graphie. Noter que si les scripteurs hésitent pour KEROUAC et pour PEREC (kérouacien et pérécien sont également attestés), ils n’hésitent pas, dans un sens, pour MAURIAC (pas de ${ }^{\circ}$ mauriaquien) ni dans l'autre pour CHIRAC (pas de ${ }^{\circ}$ chiracien) : l'application de la contrainte de registre est aussi affaire de référent. Et, on l'a dit, de discours : turciser et turquiser, québéciser et québéquiser ne se trouvent pas, d'une façon générale, dans les mêmes contextes.

La contrainte de registre, d'une façon générale, est souvent entravée par les contraintes de fidélité. Nyrop (III : 43) notait déjà : « Sous l'influence du mot primitif [...] [o]n trouve [...] autoritairisme, unitairisme, 
utilitairisme, volontairiat, à côté de autoritarisme, unitarisme, utilitarisme, volontariat ». Cette tendance est évidemment accentuée quand le locuteur est peu familier du lexique savant, et ce sont alors les gouverneurat ou sérieusité que nous avons mentionnés plus haut. Certaines de ces formes utilisent le thème $\mathrm{B}$, identique lui-même au thème A (autoritairisme, professeurat) ou différent (sérieusité), d'autres (volontairiat) une forme «savantisée » mais plus proche du thème $\mathrm{A}$ que la forme orthodoxe du thème $\mathrm{S}$. La contrainte de fidélité n'impose pas une identité intégrale avec la forme libre non marquée, elle incite à s'en rapprocher. Sur le versant populaire, l'introduction de nouvelles formes du thème B que nous avons déjà signalées parce que la contrainte de série y a sa part (CHAPEAU $\rightarrow$ chapeauter au lieu de ${ }^{\circ}$ chapeler, par exemple) ont aussi pour effet de le rapprocher du thème $\mathrm{A}$, sans qu'il soit totalement identique. D'où la formulation adoptée plus haut.

\section{$6 \quad$ Les contraintes phonologiques}

On passera plus rapidement sur les contraintes phonologiques, qui ont fait l'objet de nombreux travaux. Leur formulation, adaptée à la question qui nous occupe, pourrait être :

Contrainte de syllabation : le thème sélectionné assure au suffixe ou aux désinences verbales une accroche consonantique.

Contrainte dissimilative : le thème sélectionné évite la consécution trop rapprochée du même phonème ou de phonèmes similaires.

Contrainte prosodique : le thème sélectionné assure au dérivé un radical au moins dissyllabique.

Celle qui a le plus d'impact sur la sélection du thème est la contrainte de syllabation, ou contrainte antihiatique. La plupart des suffixes, et les désinences verbales, commencent par une voyelle et constituent la rime de la dernière syllabe du dérivé. Cette syllabe a besoin d'une attaque, qui lui est fournie par la consonne finale de la forme libre si elle en comporte une (VALLS $\rightarrow$ vallsien) ou bien, on vient de le voir, par la réalisation d'une consonne graphique interprétée comme une consonne latente (DUFLOT $\rightarrow$ duflotien). Si la graphie se termine par une voyelle, la contrainte de syllabation tend à la tronquer (TAUBIRA $\rightarrow$ taubirien) ou à susciter une épenthèse consonantique (TAUBIRA $\rightarrow$ taubiranien). La contrainte de syllabation, cependant, doit composer avec les autres contraintes, qui peuvent orienter la façon de la satisfaire ou la bloquer. Le choix entre troncation et épenthèse dépend en grande partie de la contrainte prosodique : les bases longues seront plus souvent tronquées, les bases brèves épenthésées. Le choix de la consonne est généralement commandé par le voisinage du dérivé dans la série ou dans la famille dérivationnelles: textoter rejoindra pianoter, folioter, numéroter, etc. ; sumatranais rejoint javanais et balinais ; etc. Les épenthèses les plus fréquentes devant -ien sont /s/ (à cause de l'assibilation de - $t$-) et /z/, mais sur TAUBI$\mathrm{RA} / \mathrm{n} /$ l'emporte parce que taubiranien rime léoninement avec iranien. Autres épenthèses inhabituelles suscitées par des rencontres de hasard : viragoyesque $(\leftarrow$ VIRAGO) s'aligne sur goyesque, slowler 'danser le slow' sur crawler et tarodière 'plantation de taros' sur tarauder. Hugolien, puis hugolesque, hugoliser, reprennent le thème en /1/ qui résulte d'une mécoupure de hugolâtre. Le suffixe-esque est privé d'attaque si la consonne fournie par la graphie est une sifflante (louisdeux-esque, Guy-Roux-esque), pour satisfaire à la fois la contrainte de fidélité et la contrainte dissimilative aux dépens de la contrainte de syllabation. Inversement, celle-ci pourra être satisfaite en violant la contrainte de registre : les suffixes -ier et -ette voudraient un thème populaire, mais sur PLUIE le thème savant a été préféré pour former pluvier et pluviette parce qu'il offrait une consonne.

La contrainte dissimilative (Plénat, 2011) a surtout pour effet de remplacer le suffixe attendu par un autre, ou par une variante, pour éviter une rencontre peu agréable à l'oreille. -iste a commencé à se diffuser en dehors de la sphère savante (pépiniériste) dans les cas où -ier aurait été peu euphonique ( ${ }^{\circ}$ pépiniérier). Elle a aussi un effet régressif lorsqu'elle agit sur le radical, en opposition aux autres contraintes. Sur NUIT, la contrainte phonographique et la contrainte de famille (cf. nuitée, nuitamment) voudraient que le dérivé en -ette soit ${ }^{\circ}$ nuitette. Mais /t/ est indésirable devant -ette, d'où nuisette. Symétriquement, on a vu que le thème en $/ z /$ de banlieusard pouvait difficilement être repris devant -iser, d'où banlieuriser. Devant -ité, 
l'épenthèse la plus fréquente n'est pas / $\mathrm{t} /$ mais $/ \mathrm{z} /$ : sur les adjectifs en $-u$, malgré le modèle fourni par ambiguité et quelques autres, les internautes préfèrent généralement dodusité, goulusité, poilusité, têtusité, tordusité, etc. (mais l'hiatus et/ou la semi-vocalisation redeviennent majoritaires si l'adjectif est plus long : biscornuité, farfeluité, impromptuité ${ }^{11}$. Devant -itude, en revanche, on a vu plus haut que la contrainte dissimilative est contrecarrée par la contrainte de série, qui appelle des accroches en /t/. Devant -issime, la contrainte de registre voudrait que les bases en -ique soient spirantisées, mais elle est contrecarrée par la contrainte dissimilative qui s'oppose à la consécution immédiate de deux séquences /is/. D'où LUBRIQUE $\rightarrow$ lubriquissime, au lieu de ${ }^{\circ}$ lubricissime, à côté de lubricité.

D'autres contraintes d'euphonie sont vraisemblablement à l'œuvre. On trouve par exemple, sur des bases en -art et en -ard, un certain nombre de dérivés en -aresque (mozaresque, béjaresque; godaresque, léonaresque, boulevaresque...), fidèles à la prononciation de la base mais qui violent la contrainte phonographique et surtout la puissante contrainte de série qui a suscité, symétriquement, le type BAZAR $\rightarrow$ bazarder. On peut supposer que devant -esque, qui constitue une coda branchante, une autre syllabe lourde est indésirable.

La contrainte prosodique (Plénat et Roché, 2003) a surtout pour effet d'ajouter un interfixe aux thèmes monosyllabiques pour obtenir un radical dissyllabique (PUITS $\rightarrow$ puisatier). Elle peut aussi conduire à préférer un thème savant, parce qu'il est plus long, alors que le suffixe voudrait un thème populaire : FABLE $\rightarrow$ fabulette, VIERGE $\rightarrow$ virginette, ORGUE $\rightarrow$ organette. On constate que le choix de cette solution est chaque fois renforcé par la contrainte de famille : alors que ces lexèmes n'ont pas de dérivés construits sur le thème populaire, ou qu'ils sont très peu employés, ils sont déjà dotés de dérivés plus usuels construits sur le thème savant (fabuliste, fabuler, virginal, organiste). Dans le cas de virginette, la contrainte de série joue également, puisque la sous-série en -inette est la plus fournie (avec celle en -elette) parmi les dérivés en -ette.

\section{Conclusions et perspectives}

Bien que trop sommaire, cette revue de l'allomorphie radicale des nominaux a pu montrer cependant qu'elle obéit à un certain nombre de contraintes, d'ordre lexical principalement ou dictées par la sauvegarde, phonique et graphique du lexème. Dans la mesure où la phonologie intervient, ce n'est pas sous forme de règles mais pour introduire des contraintes de «bonne formation ». La notion d'espace thématique et son application à la dérivation restent néanmoins pertinentes : on a dit pourquoi il nous semble nécessaire de distinguer, pour les nominaux comme pour les verbes, un thème présuffixal populaire et un thème présuffixal savant. Mais la sélection de l'un ou de l'autre n'est nullement automatique : elle entre dans le jeu des contraintes, comme, à l'intérieur de chaque thème, le choix des formes qui peuvent s'y trouver en concurrence.

Cette recherche, en effet, a fait apparaitre un polymorphisme plus important pour les nominaux que pour les verbes, plus important dans le lexique hérité qu'on ne le croit généralement, et autrement plus important dans les productions authentiques que dans la langue normée des dictionnaires. La masse des documents accessibles sur la Toile permet d'ouvrir toute grande la fenêtre que Frei avait entrebâillée, non pour stigmatiser des «fautes » mais pour observer la réalité de la langue. Qui est diverse, inventive, foisonnante, mais nullement anarchique : toutes les formes qui apparaissent ont leur raison d'être, et si plusieurs rivalisent pour occuper la même place c'est que ces raisons sont multiples et contradictoires.

Pour rendre compte de cette réalité, les observations et les analyses doivent évidemment être poursuivies et approfondies. Dans plusieurs directions :

- L'inscription de la dérivation dans l'espace thématique des verbes doit être précisée, comme on a essayé de le faire pour celui des nominaux. La question a déjà été bien frayée mais doit être réenvisagée sous le même éclairage. 
- La notion de contrainte doit être adaptée à l'usage qui en est fait. Il s'agit de contraintes très hétérogènes, qu'on est obligé de mettre en balance puisqu'elles sont à l'œuvre concurremment dans les faits observés. On ne peut pas se contenter de reprendre le dispositif méthodologique mis en place pour la phonologie. Il faut, par exemple, tenir compte du fait que certaines contraintes agissent positivement et d'autres négativement. La contrainte de série, parmi les premières, attire vers une forme dominante ; la contrainte dissimilative, parmi les secondes, interdit une forme dysphonique. On a vu également que le respect ou la violation d'une contrainte n'est pas un tout ou rien mais admet des degrés. Comment concevoir une pondération, un calcul de la forme finale qui tienne compte de ces fonctionnements différents?

- Il faudrait, autant que faire se peut, hiérarchiser ces contraintes. Une hiérarchisation globale, qui serait la même dans tous les cas, est probablement impossible. Mais on peut observer quels paramètres - internes ou externes - font pencher le résultat final en faveur de l'une ou de l'autre. Internes : quels complexes consonantiques, par exemple, peuvent entraîner une violation de la contrainte phonographique en faveur de la fidélité à la forme sonore, comme dans le type MOZART $\rightarrow$ mozaresque. Externes : quels types de textes, par exemple, quels contextes sociolinguistiques entraînent une violation de la contrainte de registre. Seule une collecte et une analyse de données extensives permettra d'avancer un peu dans cette voie.

- Le calcul de l'output morphophonologique doit être articulé avec celui de la construction du lexème elle-même, puisque dans le choix du suffixe ou d'un autre mode de formation d'autres contraintes sont à l'œuvre mais aussi partiellement les mêmes. Ce qui, plus encore que les tâches précédentes, suppose, parallèlement au recueil de données descriptives, une élaboration théorique dans la voie ouverte par Burzio (2002), par exemple.

\section{Références bibliographiques}

Aronoff, M. (1994). Morphology by Itself. Stems and Inflectional Classes. Cambridge (Mass.): The MIT Press.

Aronoff, M. (2007). In the beginning was the word. Language, 83.4, 803-830.

Aronoff, M. (2012). Morphological stems: what William of Ockham really said. Word Structure, 5.1, 28-51.

Bonami, O., et Boyé, G. (2003). Supplétion et classes flexionnelles dans la conjugaison du français. Langages, 152, 102-126.

Bonami, O., et Boyé, G. (2005). Construire le paradigme d'un adjectif. Recherches linguistiques de Vincennes, 34, 77-98.

Bonami, O., Boyé, G., et Kerleroux, F. (2009). L'allomorphie radicale et la relation flexion-construction. In Fradin, B., Kerleroux, F., et Plénat M. (éd.), Aperçus de morphologie du français. Saint-Denis : Presses Universitaires de Vincennes, 103-125.

Booij, G. (2010). Construction Morphology. Oxford: Oxford University Press.

Boyé, G. (2011). Régularités et classes flexionnelles dans la conjugaison du français. In Roché, M., Boyé, G., Hathout, N., Lignon, S., et Plénat, M., Des unités morphologiques au lexique. Paris / Londres : Hermès-Lavoisier, 41-68.

Boyé, G., et Plénat, M. (à paraître). L'allomorphie radicale dans les lexèmes adjectivaux du français Le cas des adverbes en -ment. In Bonet, E., Lloret, M.-R., \& Mascaró, J. (eds) Understanding allomorphy. Perspectives from Optimality Theory. London: Equinox Publishing.

Burzio, L. (2002). Surface-to-Surface Morphology: When your Representations Turn into Constraints. In Boucher, P. (ed.), Many Morphologies. Somerville: Cascadilla Press, 142-177.

Dell, F., \& Selkirk, E.O. (1978). On a Morphologically Governed Vowel Alternation in French. In Keyser, S.J. (ed.), Recent Transformational Studies in European Languages. Cambridge, MA: MIT Press, 1-51.

Frei, H. (1929). La grammaire des fautes. Genève : Slatkine reprints, 1971.

Nyrop, Kr. (1936). Grammaire historique de la langue française, tome 3 : Formation des mots. $2^{\mathrm{e}}$ éd. Copenhague : Gyldedanske Boghandel / Nordisk Forlag.

Plénat, M. (2008). Le thème L de l'adjectif et du nom. In Durand, J., Habert, B., et Laks, B. (éd.), Congrès Mondial de Linguistique Française. Paris : ILF, 1613-1626.

Plénat, M. (2009). Le conditionnement de l'allomorphie radicale en français. Mémoires de la Société de Linguistique de Paris, Nouvelle série $n^{\circ}$ 17, 119-140. 
Plénat, M. (2011). Enquête sur divers effets des contraintes dissimilatives en français. In Roché, M., Boyé, G., Hathout, N., Lignon, S., et Plénat, M., Des unités morphologiques au lexique. Paris / Londres : HermèsLavoisier, 145-190.

Plénat, M., et Plénat, C. (2011). La liaison de l'adjectif sur le nom en français : morphologie, syntaxe, phonologie. Linguistica (Ljubljana), 51, 299-315.

Plénat, M., et Roché, M. (2003). Prosodic constraints on suffixation in French. In Booij, G., DeCesaris, J., Ralli, A., \& Scalise, S. (eds.), Topics in Morphology. Selected Papers from the Third Mediterranean Morphology Meeting, Barcelona: IULA-Universitat Pompeu Fabra, 285-299.

Plénat, M., et Roché, M. (sous presse). La suffixation dénominale en -at et la loi des (sous-)séries. In Villoing, F., David, S., et Leroy, S. (éd.), Foisonnements morphologiques. Études en hommage à Françoise Kerleroux. Nanterre : Presses Universitaires de Paris Ouest.

Roché, M. (2007). Logique lexicale et morphologie : la dérivation en -isme. In Montermini, F., Boyé, G., et Hathout N. (eds), Selected Proceedings of the 5th Décembrettes: Morphology in Toulouse. Somerville: Cascadilla Press, 45-58.

Roché, M. (2010). Base, thème, radical. Recherches Linguistiques de Vincennes, 39, 95-133.

Roché, M. (2011). Pression lexicale et contraintes phonologiques dans la dérivation en -aie du français. Linguistica (Ljubljana), 51, 5-22.

Roché, M. (2013). Les alternances thématiques : morphologie ? phonologie ? lexique ? Communication au colloque La morphologie et ses interfaces, Lille, 12-13 septembre 2013.

Thornton, A.M. (2012). Reduction and maintenance of overabundance. A case study on Italian verb paradigms. Word Structure, 5.2, 183-207.

Tribout, D. (2012).Verbal stem space and verb to noun conversion in French. Word Structure, 5.1, 109-128.

Villoing, F. (2009). Les mots composés VN. In Fradin, B., Kerleroux, F., et Plénat, M., (éd.) Aperçus de morphologie du français. Saint-Denis : Presses Universitaires de Vincennes, 175-198.

\footnotetext{
${ }^{1}$ Le thème présuffixal (populaire ou savant) est également sélectionné dans les conversions $\mathrm{N} \rightarrow \mathrm{V}$ et $\mathrm{A} \rightarrow \mathrm{V}$, où il précède les désinences ou se retrouve à nu (PUITs /pui/ $\sim /$ puiz/ $\rightarrow$ nous puisons, il puise /puiz/). La composition néoclassique sélectionne également le thème présuffixal savant (suivi généralement de la voyelle de liaison $-o-$ ou $-i-$ ) pour son formant de gauche. On emploiera « thème présuffixal » pour regrouper ces trois situations.

${ }^{2}$ On appellera, pour les adjectifs, thème $\mathrm{A}$ la forme libre masculine ; thème $\mathrm{B}$ la forme présuffixale populaire, qui est aussi la forme libre féminine pour les adjectifs non réputés invariables et la forme sélectionnée pour les conversions $\mathrm{A} \rightarrow \mathrm{V}$ populaires ; thème $\mathrm{S}$ la forme présuffixale savante, sélectionnée également pour les conversions $\mathrm{A} \rightarrow \mathrm{V}$ et le premier élément des composés néo-classiques. Pour les noms, le thème $\mathrm{A}$ est celui de la forme libre, quel qu'en soit le genre ; le thème $\mathrm{B}$ celui de la suffixation et des conversions $\mathrm{N} \rightarrow \mathrm{V}$ populaires; le thème $\mathrm{S}$ celui des formations savantes.

${ }^{3} \mathrm{Au}$ fil des publications de Bonami et Boyé, la numérotation des thèmes a changé. On adoptera celle de Boyé (2011) : le thème 1 est celui du présent $1 \mathrm{P}$ et de l'imparfait; le thème 9 celui de l'infinitif; le thème 12 celui du participe passé ; le thème 13 est le «thème caché », mis en évidence par Bonami, Boyé et Kerleroux (2009), absent de la flexion et sélectionné par la dérivation savante (format- pour FORMER (formateur, formation), compress- pour COMPRIMER (compresseur, compression), etc.). On y ajoutera le thème zéro (Tribout, 2012), sélectionné pour les convers (GALOPER $\rightarrow$ galop /galo/).

${ }^{4}$ Pour ces verbes, le thème 13 et le thème zéro font problème. Nous laisserons de côté cette question.

${ }^{5}$ Cf. Plénat et Plénat (2011), qui ne l'envisagent que pour l'adjectif.

${ }^{6}$ Les deux formes sont attestées à égalité sur la Toile. D’une façon générale, les formes données ici sans astérisque ou pastille et qui ne figurent pas dans les dictionnaires ont été relevées sur la Toile, où l'on trouvera aisément références et contextes.

${ }^{7}$ La graphie est celle des journalistes qui ont rapporté les propos, d'après le site qui les commente (http://fr.wiktionary.org/wiki/Discussion utilisateur:Budelberger/D\%C3\%A9veloppement/Articles/p\%C3\%A9ren).

On ne sait comment le locuteur écrirait ces adjectifs, s'il écrivait.

${ }^{8}$ Plus précisément : il n'y a plus (aveuer est devenu avouer, baer est devenu béer (ou bayer), etc.).

${ }^{9}$ Pour une description plus précise : Plénat, 2008.

${ }^{10}$ Certains doublets viennent du fait que la forme savante a été empruntée au latin, mais d'autres sont bien des formations françaises.
} 


\footnotetext{
${ }^{11}$ Exemples empruntés à la base de données MORDAN d'Aurore Koehl. ttps://sites.google.com/site/koehlaurore/these/CORPUS_THESE_KOEHL_A.xlsx?attredirects $=0 \& \mathrm{~d}=1$
} 